\title{
Erratum to: Comparative codon usage between the three main viruses in pestivirus genus and their natural susceptible livestock
}

\author{
Jian-hua Zhou $\cdot$ Zong-liang Gao $\cdot$ Jie Zhang $\cdot$ \\ Hao-tai Chen $\cdot$ Zygmunt Pejsak $\cdot$ Li-na Ma \\ Yao-zhong Ding $\cdot$ Yong-sheng Liu
}

Published online: 30 May 2012

(C) Springer Science+Business Media, LLC 2012

\section{Erratum to: Virus Genes (2012) 44:475-481 \\ DOI 10.1007/s11262-012-0731-z}

\begin{abstract}
A language issue has been noted in the title of the published article. Instead of "Comparative the codon usage between the three main viruses in pestivirus genus and their natural susceptible livestock", it should be "Comparative codon usage between the three main viruses in pestivirus genus and their natural susceptible livestock".
\end{abstract}

The online version of the original article can be found under doi:10.1007/s11262-012-0731-z.

J. Zhou · Z. Gao · J. Zhang $\cdot$ H. Chen $\cdot$ L. Ma $\cdot$ Y. Ding

Y. Liu ( $\square)$

State Key Laboratory of Veterinary Etiological Biology,

National Foot-and-Mouth Disease Reference Laboratory,

Lanzhou Veterinary Research Institute, Chinese Academy

of Agricultural Sciences, Lanzhou 730046, Gansu,

People's Republic of China

e-mail: liuyongshengvip8@163.com

J. Zhou

e-mail: zhoujianhuazjh@163.com

\section{Z. Pejsak}

Department of Swine Diseases, National Veterinary Research

Institute, 57 Partyzantow, 24-100 Puławy, Poland 